\title{
3-Hydroxypropylmercapturic Acid
}

National Cancer Institute

\section{Source}

National Cancer Institute. 3-Hydroxypropylmercapturic Acid. NCI Thesaurus. Code C107144.

A modified form of acetylcysteine that is a metabolite of acrolein and may be found in the urine of smokers. 